\title{
Variáveis sociodemográficas e os consumidores de alimentos orgânicos no Brasil
}

\author{
Sociodemographic Variables and Consumers of Organic Food in Brazil
}

\begin{abstract}
Gabriel Horn Iwaya
Andrea Valéria Steil ${ }^{2}$

\section{Resumo}

Em paralelo ao crescimento do mercado de alimentos orgânicos, crescem as pesquisas que investigam quem são os consumidores de alimentos orgânicos. Essa pesquisa foi elaborada com o objetivo de verificar a existência de associações entre variáveis sociodemográficas e grupos de consumidores de alimentos convencionais e orgânicos. Foi utilizado o método de levantamento com amostragem não probabilística e coleta de dados online. A análise de dados utilizou técnicas estatísticas descritivas e de associação. Os resultados revelaram a inexistência de associações significativas entre as variáveis sociodemográficas sexo, faixa etária, escolaridade, renda, estado civil e os grupos de consumidores de alimentos convencionais e orgânicos. Estudos futuros podem considerar investigar fatores psicológicos (crenças, percepções, atitudes) dos consumidores com relação ao consumo de alimentos orgânicos.

Palavras-chave: Perfil do Consumidor; Psicologia do Consumidor; Comportamento do Consumidor..

Abstract

Simultaneously with the growth of the organic food market, the number of researchers seeking to investigate "Who are the consumers of organic food?" is growing. This research was carried out to verify associations between sociodemographic variables and groups of food consumers (conventional or organic). The survey method with non-probabilistic sampling and online data collection was used. Data analysis used descriptive and association statistical techniques (Pearson's chi-square). The results did not reveal significant associations between the sociodemographic variables (sex, age group, education, income, marital status) and consumer groups. Future studies may consider investigating consumers' psychological factors (beliefs, perceptions, attitudes) regarding organic foods' consumption.
\end{abstract}

Keywords: Consumer Profile; Consumer Psychology. Consumer Behavior.

\footnotetext{
1 gabrieliwaya@hotmail.com, Universidade Federal de Santa Catarina - Florianópolis/SC [Brasil] DORCID Id: http://orcid.org/0000-0002-1075-9294

2 andrea.steil@ufsc.b, Universidade Federal de Santa Catarina - Florianópolis/SC [Brasil] (1)ORCID Id: http://orcid.org/0000-0001-7853-6532
} 


\section{Introdução}

O último relatório produzido pelo Research Institute of Organic Agriculture, em parceria com o International Federation of Organic Agriculture Movements (IFOAM), apresenta dados globais sobre a agricultura orgânica mundial. O relatório, intitulado "The World of Organic Agriculture: Statistics and Emerging Trends 20I9", reporta informações sobre o crescimento da cadeia de produção de alimentos orgânicos. Os dados desse relatório anual são relativos aos resultados obtidos até o período de dois anos atrás, portanto, de 20I7 (Willer \& Lernoud, 20I9a).

De acordo com o relatório, em 2017 foram destinados 69,8 milhões de hectares de terras ao plantio de orgânicos, o que representa um aumento de $20 \%$ em relação a 20 o. O número de agricultores também aumentou $5 \%$ nesse período, alcançando a marca de 2,9 milhões de produtores em 2017 (Willer, Lernoud, \& Kemper, 20I9). O faturamento do mercado global de produtos orgânicos aumentou $7,7 \% \mathrm{em} 20 \mathrm{O}$ em relação ao ano anterior, atingindo novo recorde (97 bilhões de dólares). Nos últimos seis anos (2OII 2OI7), o faturamento do mercado de produtos orgânicos cresceu $46 \%$, mantendo a média de crescimento anual de 7,6\% (Sahota, 20I8; 2OI9).

No Brasil, a venda de produtos orgânicos (varejo e exportação) alcançou um faturamento de aproximadamente 995 milhões de dólares em 2016 (último valor contabilizado no relatório de Willer e Lernoud (2orgb)). O mercado de produtos orgânicos no Brasil envolveu a participação de mais de I5 mil produtores e destinou cerca de I,I milhão de hectares de terras ao seu cultivo em 20I7 (Willer \& Lernoud, 2OI9b).

Em paralelo ao contínuo aumento do mercado de alimentos orgânicos, cresceu nas últimas décadas o número de pesquisas que procuram investigar quem são os consumidores de alimentos orgânicos. Respostas para essa questão são de grande valor estratégico para os sistemas de produção e comercialização de alimentos orgânicos, pois fornecem evidências sobre o perfil do consumidor. Por sua vez, este conhecimento facilita o desenvolvimento de processos de segmentação de mercado e a tomada de decisão nas organizações (Pearson, Henryks, \& Jones, 2OII).

Há um predomínio de estudos com variáveis sociodemográficas para a identificação dos consumidores que compram ou não compram alimentos orgânicos. Entretanto, de acordo com Pearson, Henryks e Jones (2OII), os resultados obtidos nos últimos $2 \mathrm{O}$ anos de pesquisa nesse sentido apontam para a inexistência de associações entre variáveis sociodemográficas e os grupos de consumidores de alimentos convencionais e orgânicos. As pesquisas indicam que os consumidores de alimentos orgânicos estão distribuídos em todos os segmentos sociodemográficos e que a análise de variáveis desse tipo tem apresentado resultados contraditórios. Apesar deste aspecto, a literatura internacional aponta para algumas tendências, como: a) serem pessoas do sexo feminino; b) de faixa etária mais elevada; c) com maior nível de escolaridade; e d) com maior renda (Hughner, Mcdonagh, Prothero, Shultz, \& Stanton, 2007; Pearson, Henryks, \& Jones, 2OII).

Pesquisas com consumidores dos Estados Unidos, que responde por cerca da metade do faturamento global do mercado de orgânicos (Sahota, 20I9), chegaram a conclusões similares em alguns aspectos. Por exemplo, o estudo de Dettmann e Dimitri (2009) concluiu que o nível de escolaridade e da renda familiar dos consumidores são 
fatores que influenciam positivamente a probabilidade de estes comprarem alimentos orgânicos. Outro estudo, conduzido por Dimitri e Dettmann (2012), chegou à conclusão de que pessoas casadas apresentam maior probabilidade de comprar alimentos orgânicos. Esse estudo também identificou que a probabilidade de comprar alimentos orgânicos aumenta com o aumento da renda e da escolaridade dos consumidores.

Com relação ao perfil do consumidor de alimentos orgânicos no Brasil, destaca-se o estudo bibliométrico de Souza, Filho, Santos, Melo e Cavalcanti (2018). O estudo analisou 24 artigos nacionais, com amostras de consumidores de alimentos orgânicos coletadas em feiras públicas e sessões de supermercados destinadas à comercialização desse segmento de produtos de cinco regiões brasileiras, com predominância para as regiões Sul e Sudeste, a saber: Norte (or); Nordeste (o5); Centro-Oeste (o3); Sudeste (o7); Sul (o8). De acordo com Souza et al. (2018), o perfil do consumidor de alimentos orgânicos no Brasil é o de uma pessoa do sexo feminino, casada, com idade superior a 30 anos, ensino superior completo e renda elevada.

Destaca-se que o trabalho de Souza et al. (2018) traça seus resultados com base em prevalências encontradas nas amostras de cada pesquisa analisada. Ou seja, com base no número de determinados casos dividido pelo número de participantes da amostra e multiplicado por Ioo (ex. número de mulheres de uma amostra divido pelo número de participantes da amostra vezes IOO). Entretanto, a prevalência de uma característica em uma amostra (muitas vezes obtida por conveniência) não significa que exista uma associação entre essa característica e o fato desse consumidor se denominar comprador de alimentos orgânicos ou de alimentos convencionais. Pelo contrário, isso pode apenas nos informar que mulheres com faixa etária acima de 30 anos e níveis elevados de renda e escolaridade sejam pessoas mais dispostas a doar seu tempo para responder uma pesquisa.

Dessa forma, o objetivo desta pesquisa é o de verificar a existência de associações entre variáveis sociodemográficas e os grupos de consumidores brasileiros de alimentos convencionais ou de alimentos orgânicos. Como apontam Dettmann (2008) e Pearson, Henryks e Jones (2OII), os resultados de pesquisas internacionais ainda apresentam resultados conflitantes e insuficientes para a determinação de um perfil consistente do "típico" consumidor de alimentos orgânicos.

Embora não exista um consenso entre as pesquisas internacionais, os trabalhos de Hughner et al. (2007), Dettmann e Dimitri (2009), Pearson, Henryks e Jones (2OII), e Dimitri e Dettmann (2OI2) apresentam evidências sobre a existência de associações entre variáveis sociodemográficas e os consumidores de alimentos orgânicos. As evidências dos trabalhos mencionados apontam que os consumidores de alimentos orgânicos apresentam maior probabilidade de serem: a) do sexo feminino; b) com maior faixa etária; c) maior nível de escolaridade; d) maior renda; e) casados. Dessa forma, a presente pesquisa propôs como ponto de partida as seguintes hipóteses, para verificar se elas são verdadeiras na realidade brasileira:

Hr: Existe associação entre o sexo e a distribuição dos consumidores entre os grupos de consumidores de alimentos convencionais ou alimentos orgânicos.

$\mathrm{H}_{2}$ : Existe associação entre a faixa etária e a distribuição dos consumidores entre os grupos de consumidores de alimentos convencionais ou alimentos orgânicos. 
$\mathrm{H}_{3}$ : Existe associação entre o nível de escolaridade e a distribuição dos consumidores entre os grupos de consumidores de alimentos convencionais ou alimentos orgânicos.

$\mathrm{H}_{4}$ : Existe associação entre o nível de renda e a distribuição dos consumidores entre os grupos de consumidores de alimentos convencionais ou alimentos orgânicos.

$\mathrm{H}_{5}$ : Existe associação entre o estado civil e a distribuição dos consumidores entre os grupos de consumidores de alimentos convencionais ou alimentos orgânicos.

A seção subsequente apresentará o detalhamento metodológico utilizado para o alcance do objetivo proposto por esta pesquisa: verificar a existência de associações entre variáveis sociodemográficas e os grupos de consumidores brasileiros de alimentos convencionais ou de alimentos orgânicos. Essa pesquisa pretendeu encontrar respostas para as questões relacionadas ao delineamento de um perfil do consumidor de alimentos orgânicos. Mais especificamente, procurou responder a seguinte questão: "Quem são os consumidores de alimentos orgânicos?”.

\section{Método}

\section{Delineamento e participantes}

Esta é uma pesquisa quantitativa, com delineamento de levantamento e aplicação de questionário online como instrumento de coleta de dados. O desenho do levantamento foi de corte transversal, com obtenção da amostra em um determinado momento. A pesquisa partiu de uma abordagem amostral não probabilística, por conveniência (Shaughnessy, Zechmeister, \& Zechmeister, 20I2; Sampieri, Collado, \& Lucio, 2OI3).

Foram estabelecidos dois critérios para a participação na pesquisa: a) que o participante tivesse idade superior a I8 anos; e b) que o participante fosse responsável ou parcialmente responsável pelas compras de alimentos de sua residência.

\section{Instrumento de coleta de dados}

O instrumento utilizado na pesquisa foi um questionário online dividido em quatro sessões: I) de apresentação do Termo de Consentimento Livre e Esclarecido (TCLE) para participação na pesquisa; 2) de triagem dos participantes com a apresentação do critério de inclusão da pesquisa; 3 ) de itens sociodemográficos da pesquisa;4) com item de mensuração de comportamento passado por autorrelato. A coleta de dados foi iniciada em 28/o5/2019 e se estendeu até o8/o7/2019.

$\mathrm{Na}$ sessão relativa às variáveis sociodemográficas, os participantes foram solicitados a responder questões relacionadas: I) ao sexo biológico (feminino/masculino); 2) à idade (em anos completos); 3 ) ao nível de escolaridade (concluída); 4) ao estado civil; 5) à renda familiar; 6) ao estado onde mora; e 7) à cidade onde mora. Todas as questões foram configuradas para que fossem de caráter obrigatório, não sendo possível concluir a submissão do questionário sem responder a todas as questões.

A sessão relativa ao comportamento passado contou com apenas um item. $\mathrm{O}$ item foi apresentado por meio de uma afirmação direta com possibilidade de resposta 
dicotômica, a saber: "Eu me considero um consumidor de alimentos orgânicos" ( $\operatorname{sim} /$ não).

\section{Cuidados éticos}

Essa pesquisa adotou, em seu planejamento e sua execução, o que determina a resolução $n^{\circ}$ 5IO/I6 do Conselho Nacional de Saúde (CNS), voltada aos preceitos éticos e da proteção aos participantes da pesquisa. A execução da coleta de dados dessa pesquisa foi condicionada à aprovação do Comitê de Ética em Pesquisa com Seres Humanos (CEPSH-UFSC). O parecer consubstanciado de aprovação da pesquisa pelo CEPSH pode ser verificado junto a Plataforma Brasil pelo número: 3.027.720.

\section{Procedimentos de análise de dados}

Os dados coletados foram analisados por meio de técnicas estatísticas: descritivas (dados sociodemográficos) e de associação (qui-quadrado de Pearson). Para os procedimentos de análise foi utilizado o software IBM SPSS Statistics 25.o.

A análise estatística descritiva das variáveis sociodemográficas foi feita por meio da contagem de frequências e percentuais. A condução do teste de associação (quiquadrado de Pearson) foi feita com a utilização do comando de tabulação cruzada, considerando a distribuição das variáveis sociodemográficas em função da variável dicotômica "Eu me considero um consumidor de alimentos orgânicos" (sim/não). O nível de significância estabelecido foi de $95 \%$.

\section{Resultados}

A pesquisa contou com a participação de 705 consumidores de 23 unidades federativas (estados) do Brasil, distribuídos entre i6 5 municípios. A grande maioria dos participantes reside nas regiões Sul $(76,74 \%)$ e Sudeste (I6,31\%), sendo que o maior número de participações foi de consumidores residentes nos municípios de Joinville (38,30\%), Florianópolis (I $4,60 \%)$, São Paulo (3,54\%) e Curitiba (3,26\%). A frequência dos participantes por unidade federativa pode ser observada na Tabela I. 


\begin{tabular}{lcc}
\hline Tabela 1 & \multicolumn{3}{c}{} \\
Frequência dos participantes por unidade federativa & $(N=705)$ \\
\hline Estado onde mora & Frequência & Percentagem \\
Santa Catarina & 465 & $65,96 \%$ \\
São Paulo & 69 & $9,79 \%$ \\
Paraná & 43 & $6,10 \%$ \\
Rio Grande do Sul & 33 & $4,68 \%$ \\
Rio de Janeiro & 28 & $3,97 \%$ \\
Minas Gerais & 16 & $2,27 \%$ \\
Goiás & 10 & $1,42 \%$ \\
Bahia & 8 & $1,13 \%$ \\
Pernambuco & 5 & $0,71 \%$ \\
Roraima & 4 & $0,57 \%$ \\
Distrito Federal & 3 & $0,43 \%$ \\
Paraíba & 3 & $0,43 \%$ \\
Alagoas & 2 & $0,28 \%$ \\
Amapá & 2 & $0,28 \%$ \\
Espírito Santo & 2 & $0,28 \%$ \\
Mato Grosso & 2 & $0,28 \%$ \\
Mato Grosso do Sul & 2 & $0,28 \%$ \\
Rio Grande do Norte & 2 & $0,28 \%$ \\
Rondônia & 2 & $0,28 \%$ \\
Amazonas & 1 & $0,14 \%$ \\
Pará & 1 & $0,14 \%$ \\
Piauí & 1 & $0,14 \%$ \\
Sergipe & 1 & $0,14 \%$ \\
Total & $\mathbf{1 0 5}$ & $\mathbf{1 0 0 \%}$ \\
\hline
\end{tabular}

Fonte: Autores, 2020.

O perfil sociodemográfico dos participantes caracterizou-se pela prevalência de pessoas do sexo feminino $(67,40 \%)$, de faixa etária entre $28-37$ anos $(30,78 \%)$, com ensino superior completo $(37,60 \%)$, casados(as) ou em união estável $\left(5^{1}, 60 \%\right)$ e renda familiar entre 3 e 6 salários mínimos $(26,24 \%)$. O perfil sociodemográfico com os valores específicos de frequências e percentuais pode ser observado na Tabela 2. 

orgânicos no Brasil. RAD - Revista Administração em Diáloqo, 23(1), 54-66.

Tabela 2

Perfil sociodemográfico dos participantes da pesquisa $(N=705)$

Variáveis sociodemográficas

Frequências Percentagem

Sexo

Feminino

475

67,4

Masculino

\section{Faixa etária}

$18-27$ anos

$28-37$ anos

$38-47$ anos

$48-57$ anos

16,45

Acima de 58

Nível de escolaridade

Ensino médio ou inferior

$\begin{array}{cc}116 & 16,45 \\ 70 & 9,93\end{array}$

Ensino superior

Pós-graduação (especialização)

Mestrado

Doutorado

\section{Estado civil}

Casado(a)/União estável 364

Solteiro(a)

Divorciado(a)/Separado(a) ou Viúvo(a)

\section{Renda familiar}

Até 3 salários mínimos

Entre 3 e 6 salários mínimos

Entre 6 e 9 salários mínimos

Entre 9 e 12 salários mínimos

Entre 12 e 15 salários mínimos

Fonte: Autores, 2020.

A contagem de frequências e percentuais do perfil sociodemográfico dos participantes da pesquisa foi novamente feita, dividindo-se a base de dados em dois grupos. A divisão foi feita para distinguir o perfil dos consumidores de acordo com a variável dicotômica "Eu me considero um consumidor de alimentos orgânicos" (sim/não). Em sequência, foi feita a tabulação cruzada das variáveis e a aplicação do teste de associação qui-quadrado de Pearson, com $5 \%$ de significância. Entretanto, as variáveis "faixa etária", "nível de escolaridade" e "estado civil” apresentaram contagem mínima esperada menor do que cinco, por isso tiveram seus níveis categóricos recodificados.

Em faixa etária, os casos pertencentes às categorias “ $58-67$ anos" e " $68-77$ anos" foram aglutinados como "acima de $5^{8}$ anos". Em nível de escolaridade, os casos pertencentes às categorias "ensino fundamental" e "ensino médio" foram aglutinados como "ensino médio ou inferior". Em estado civil, os casos pertencentes às categorias 
“divorciado(a)/separado(a)" e "viúvo(a)", foram aglutinados como “divorciado(a)/separado(a) ou viúvo(a)".

Após a recodificação dos níveis categóricos das variáveis sociodemográficas, a contagem de frequências e percentuais do perfil sociodemográfico dos participantes da pesquisa foi refeita. Em sequência, foi feita a tabulação cruzada das variáveis e a aplicação do teste de associação qui-quadrado de Pearson, com 5\% de significância. Todas as variáveis apresentaram valores esperados maiores do que cinco nessa nova recodificação. O segundo perfil sociodemográfico dos participantes da pesquisa e as evidências estatísticas de relação entre as variáveis podem ser observadas na Tabela 3.

\begin{tabular}{|c|c|c|c|c|c|c|c|}
\hline \multicolumn{8}{|c|}{$\begin{array}{l}\text { Tabela } 3 \\
\text { Perfil sociodemográfico dos participantes de acordo com a variável di } \\
\text { "Eu me considero um consumidor de alimentos orgânicos" (sim/não) }\end{array}$} \\
\hline \multirow{3}{*}{ Variáveis sociodemográficas } & \multicolumn{4}{|c|}{$\begin{array}{l}\text { "Eu me considero um consumidor } \\
\text { de alimentos orgânicos" }\end{array}$} & \multirow{2}{*}{\multicolumn{3}{|c|}{$\begin{array}{l}\text { Qui-quadrado de } \\
\text { Pearson }\end{array}$}} \\
\hline & \multicolumn{2}{|c|}{ Não (N=468) } & \multicolumn{2}{|c|}{$\operatorname{Sim}(N=237)$} & & & \\
\hline & $\mathbf{N}$ & $\%$ & $\mathbf{N}$ & $\%$ & $\chi^{2}$ & (gl) & p-valor \\
\hline \multicolumn{8}{|l|}{ Sexo } \\
\hline Feminino & 316 & 67,52 & 159 & 67,09 & 0,134 & -1 & 0,908 \\
\hline Masculino & 152 & 32,48 & 78 & 32,91 & & & \\
\hline \multicolumn{8}{|l|}{ Faixa etária } \\
\hline $18-27$ anos & 112 & 23,93 & 41 & 17,3 & 4,943 & -4 & 0,293 \\
\hline $28-37$ anos & 139 & 29,7 & 78 & 32,91 & & & \\
\hline $38-47$ anos & 97 & 20,73 & 52 & 21,94 & & & \\
\hline $48-57$ anos & 72 & 15,38 & 44 & 18,57 & & & \\
\hline Acima de 58 anos & 48 & 10,26 & 22 & 9,28 & & & \\
\hline \multicolumn{8}{|l|}{ Nível de es colaridade } \\
\hline Ensino médio ou inferior & 56 & 11,97 & 36 & 15,19 & 9,261 & -4 & 0,054 \\
\hline Ensino superior & 185 & 39,53 & 80 & 33,76 & & & \\
\hline Pós-graduação (especialização) & 126 & 26,92 & 65 & 27,43 & & & \\
\hline Mestrado & 69 & 14,74 & 27 & 11,39 & & & \\
\hline Doutorado & 32 & 6,84 & 29 & 12,24 & & & \\
\hline \multicolumn{8}{|l|}{ Estado civil } \\
\hline Casado(a)/União estável & 234 & 50 & 130 & 54,85 & 4,936 & -2 & 0,085 \\
\hline Solteiro(a) & 184 & 39,32 & 74 & 31,22 & & & \\
\hline Divorciado(a)/Separado(a)/Viúvo(a) & 50 & 10,68 & 33 & 13,92 & & & \\
\hline \multicolumn{8}{|l|}{ Renda familiar } \\
\hline Até 3 salários mínimos & 81 & 17,31 & 48 & 20,25 & 7,574 & -5 & 0,181 \\
\hline Entre 3 e 6 salários mínimos & 133 & 28,42 & 52 & 21,94 & & & \\
\hline Entre 6 e 9 salários mínimos & 113 & 24,15 & 54 & 22,78 & & & \\
\hline Entre 9 e 12 salários mínimos & 57 & 12,18 & 25 & 10,55 & & & \\
\hline Entre 12 e 15 salários mínimos & 44 & 9,4 & 27 & 11,39 & & & \\
\hline Acima de 15 salários mínimos & 40 & 8,55 & 31 & 13,08 & & & \\
\hline
\end{tabular}

Fonte: Autores, 2020.

As evidências encontradas não revelaram níveis de significância aceitáveis $(\mathrm{p}<0,05)$ que suportem a hipótese alternativa de associação entre as variáveis 
sociodemográficas e os grupos de consumidores de alimentos (orgânicos ou convencionais). Reteve-se, portanto, a hipótese nula, de que não há associação entre tais variáveis e os grupos de consumidores. O resumo dos resultados que apontam para a rejeição das hipóteses estabelecidas nesta pesquisa pode ser observado na Tabela 4.

Tabela 4
Avaliação das hipóteses estabelecidas pela pesquisa

H1. Existe associação entre o sexo e a distribuição dos

consumidores entre os grupos de consumidores de

alimentos convencionais ou alimentos orgânicos.

H2. Existe associação entre a faixa etária e a distribuição dos consumidores entre os grupos de consumidores de alimentos convencionais ou alimentos orgânicos.

H3. Existe associação entre o nível de escolaridade e a distribuição dos consumidores entre os grupos de consumidores de alimentos convencionais ou alimentos orgânicos.

H4. Existe associação entre o nível de renda e a distribuição dos consumidores entre os grupos de consumidores de alimentos convencionais ou alimentos orgânicos.

H5. Existe associação entre o estado civil e a distribuição dos consumidores entre os grupos de consumidores de alimentos convencionais ou alimentos orgânicos.

\begin{tabular}{cccc}
\multicolumn{2}{c}{ Qui-quadrado de Pearson } & \multirow{2}{*}{ Decisão } \\
\hline$\chi^{2}$ & (gl) & p-valor & \\
0,134 & -1 & 0,908 & Rejeitar
\end{tabular}

$\begin{array}{llll}4,943 & -4 & 0,293 \quad \text { Rejeitar }\end{array}$

$9,261 \quad-4 \quad 0,054 \quad$ Rejeitar

$7,574 \quad-5 \quad 0,181 \quad$ Rejeitar

$\begin{array}{llll}4,936 & -2 & 0,085 & \text { Rejeitar }\end{array}$

Fonte: Autores, 2020.

\section{Discussão}

As evidências obtidas nessa pesquisa não suportam as hipóteses de existência de associações entre as variáveis sociodemográficas dos participantes (sexo, faixa etária, nível de escolaridade, estado civil, renda familiar) e a variável de resposta dicotômica "Eu me considero um consumidor de alimentos orgânicos" ( $\operatorname{sim} /$ não). Esses achados estão de acordo com as revisões da literatura internacional feitas por Hughner et al. (2007) e Person, Henryks e Jones (2OII) e contrariam as tendências apontadas por Souza et al. (20I8) em sua revisão da literatura nacional.

Souza et al. (2018) destacam que fatores demográficos, como gênero feminino, idade superior a trinta anos, ensino superior completo e renda elevada conformam uma forte tendência do perfil do consumidor de alimentos orgânicos no mercado nacional. A análise das variáveis sociodemográficas desse estudo não apresentou evidências que suportem essa afirmação. Isso significa que, para os respondentes deste estudo, não há justificativa para a utilização de variáveis sociodemográficas como alternativa para a segmentação dos consumidores de alimentos orgânicos.

Outro estudo, realizado por Chen, Lobo e Rajendran (20I4), também não encontrou relação significativa entre as variáveis "gênero", "idade" e "escolaridade" e a intenção de compra de alimentos orgânicos. A exceção destacada pelo estudo de Chen, Lobo e Rajendran (20I4) foi a variável renda, que demonstrou exercer efeito significativo $(\mathrm{r}=\mathrm{O}, \mathrm{I} 39 ; \beta=\mathrm{O}, \mathrm{I} 43 ; \mathrm{p}<\mathrm{O}, \mathrm{OOI})$ como preditora da intenção de compra de alimentos 
orgânicos. Chen, Lobo e Rajendran (20I4) realizaram a pesquisa com consumidores chineses, utilizando amostragem por conveniência $(n=907)$. A análise estatística foi feita por meio da técnica de regressão linear múltipla hierárquica. A análise resultou em um modelo estatisticamente significativo $\left[\mathrm{F}\left(2,9^{12}\right)=25^{8.78} ; \mathrm{p}<\mathrm{O}, \mathrm{OOI} ; \mathrm{R}^{2}=0,373\right]$. Entretanto, o conjunto de variáveis atitudinais respondeu pela maior parcela da variância explicativa do modelo $(35 \%)$, enquanto a variável renda respondeu por apenas $2 \%$ da variância.

\section{Limitações e estudos futuros}

Toda pesquisa possui algum tipo de limitação. Uma limitação metodológica dessa pesquisa diz respeito ao tipo de amostragem utilizado. A abordagem amostral utilizada por essa pesquisa foi não-probabilística (obtida por conveniência) e a coleta foi realizada via online, o que não garante que cada elemento da população brasileira tenha recebido igual chance de ser incluído na amostra. A escolha pela abordagem amostral não-probabilística deu-se em virtude das condições temporais e financeiras limitadas para execução do estudo. Apesar da agilidade e do baixo custo da utilização de redes sociais para a pesquisa, há a possibilidade de viés dos respondentes - por exemplo, devido às disparidades sociodemográficas da população investigada ( $33,8 \%$ dos participantes com nível superior, 5I\% com pós-graduação, mestrado ou doutorado).

De acordo com Shaughnessy, Zechmeister e Zechmeister (20I2), a abordagem amostral não-probabilística costuma gerar amostras com viés de seleção, causando superrepresentação de um segmento da população ou, do contrário, causando subrepresentação de outro segmento populacional. Dessa forma, é necessário destacar que a amostra obtida por essa pesquisa não é representativa da população brasileira. Como alternativa para sanar essa limitação, pesquisas futuras podem considerar a viabilidade da utilização de abordagens amostrais representativas (aleatória ou aleatória estratificada) da população brasileira.

Os resultados obtidos por essa pesquisa não suportaram as hipóteses de associação entre as variáveis sociodemográficas e os grupos de consumidores de alimentos (convencionais ou orgânicos). Em suas discussões, Person, Henryks e Jones (2OII) destacam que estudos futuros devem considerar novos caminhos para identificar "Quem são os consumidores de alimentos orgânicos?”. Entre as possibilidades de pesquisa, os autores recomendam investigações que levem em consideração a função que os alimentos orgânicos desempenham na vida dos consumidores e o contexto em que o comportamento de compra de alimentos orgânicos é realizado.

Hughner et al. (2007) recomendam que estudos futuros sejam desenvolvidos considerando-se a investigação de fatores motivacionais, perceptivos e atitudinais dos consumidores com relação ao consumo de alimentos orgânicos. Os autores sugerem, também, que sejam conduzidas pesquisas com abordagem mais "psicográfica” e/ou holística, que considerem a investigação dos valores e dos estilos de vida dos consumidores de alimentos orgânicos. De acordo com Hughner et al. (2OOZ), as pesquisas futuras devem considerar métodos de pesquisa interpretativos como alternativa, com os objetivos de descrever os motivos subjacentes que conduzem esse processo de tomada de decisão.

\section{Revista Administração em Diálogo}

ISSN 2178-0080

PUC-SP 
Estudos com o objetivo de explicar padrões comportamentais que orientam os processos de consumo e de estilos de vida dos indivíduos de uma determinada sociedade são fundamentais. Isto porque os padrões comportamentais dos indivíduos de uma sociedade exercem impactos diretos e indiretos, tanto no meio ambiente como no bemestar e na qualidade de vida de todos. Não por menos, as investigações de pesquisa e iniciativas relacionadas ao desenvolvimento e estudo de processos de consumo responsável (sustentável, verde, etc.) tornaram-se de interesse internacional (Nações Unidas, 20I5; Groening, Sarkis, \& Zhu, 20I8).

A investigação de um processo de consumo pode utilizar diferentes arcabouços teóricos que engendram, de forma explícita ou implícita, "modelos” explicativos do comportamento do consumidor. Modelos teóricos fornecem estruturas heurísticas úteis para conceituar, caracterizar e explorar diferentes tipos de consumo e consumidores (Jackson, 2005).

A Teoria do Comportamento Planejado (TCP), proposta por Icek Ajzen (1985), se destaca como um dos modelos teóricos mais utilizados por pesquisas com o objetivo de investigar comportamentos de consumo sustentável (Peattie, 2OIO; Joshi \& Rahman, 2OI5; Liobikienè \& Bernatonienè, 2OI7). Em comparação com outros modelos, a TCP também tem apresentado melhor poder preditivo (Bamberg \& Schmidt, 2003), inclusive no caso de pesquisas voltadas ao consumo sustentável (Kaiser, Hübner, \& Bogner, 2005).

A título de exemplo, podem ser destacados os trabalhos de revisão sistemática e meta-análise de Han e Stoel (20I6) e Scalco, Noventa, Sartori e Ceschi (20I7), que avaliaram uma série de estudos voltados à compreensão da intenção de compras responsáveis, utilizando a TCP. Essas revisões tiveram por objetivo investigar o efeito dos preditores da TCP na formação da intenção de compra de "produtos socialmente responsáveis" e "alimentos orgânicos”, respectivamente. Em sua conclusão, Scalco et al. (20I7, p. 236) destacam que "a TCP representa uma sólida estrutura psicológica que, mais do que outras, conseguiu desvelar as principais motivações por trás das escolhas alimentares em relação ao consumo sustentável".

O desenvolvimento de pesquisas futuras utilizando o modelo da TCP para a investigação do comportamento de compra de alimentos orgânicos no Brasil é compreendido como uma alternativa potencialmente promissora que vem apresentando evidências consistentes em nível internacional. Nesse sentido, o relatório de Jackson (2005) também fornece uma revisão sobre outros diversos modelos explicativos do comportamento do consumidor que são utilizados internacionalmente em investigações voltadas a processos de consumo responsável. Tal rol de modelos também pode ser útil como alternativa para o empreendimento de futuras investigações, quantitativas e qualitativas, voltadas aos consumidores de alimentos orgânicos brasileiros.

\section{Financiamento}

O presente trabalho foi realizado com apoio da Coordenação de Aperfeiçoamento de Pessoal de Nível Superior - Brasil (CAPES) - Código de Financiamento oor. 


\section{Referèncias}

Bamberg, S., \& Schmidt, P. (2003). Incentives, morality, or habit? Predicting students' car use for university routes with the models of Ajzen, Schwartz, and Triandis. Environment and Behavior, 35(2), p. 264-285. https://doi.org/IO.II77/OOI39I6502250I34

Chen, J., Lobo, A., \& Rajendran, N. (20I4). Drivers of organic food purchase intentions in mainland China-evaluating potential customers' ${ }^{\prime}$ attitudes, demographics and segmentation. International Journal of Consumer Studies, 38(4), p. 346-356. https://doi.org/IO.III/ ijcs.I2O95

Dalcin, D., Souza, A. R. L., Freitas, J. B., Padula, A. D., \& Dewes, H. (2OI4). Organic products in Brazil: From an ideological orientation to a market choice. British Food Journal, II6(г2), p. I998-2OI5. https://doi.org/IO.IIO8/BFJ-OI-2OI3-OOO8

Dettmann, R. L. (2008). Organic Produce: Who's Eating it? A Demographic Profile of Organic Produce Consumers. Orlando, Florida: American Agricultural Economics Association Annual Meeting. https://doi.org/I0.22004/ag.econ.6446

Dettmann, R. L., \& Dimitri, C. (2009). Who's buying organic vegetables? Demographic characteristics of US consumers. Journal of Food Products Marketing, I6(I), p. 79-9I. https://doi.org/IO.IO80/IO4544409O34I57O9

Dimitri, C., \& Dettmann, R. L. (20I2). Organic food consumers: what do we really know about them? British Food Journal, II4(8), p. II57-II83. https://doi.org/ıo.IIO8/oOO7О7ОI2II25 2IOI

Groening, C., Sarkis, J., \& Zhu, Q. (2018). Green marketing consumer-level theory review: A compendium of applied theories and further research directions. Journal of Cleaner Production, I72(I), p. I848-I866. https://doi.org/IO.IOI6/j.jclepro.2OI7.I2.0O2

Han, T., \& Stoel, L. (2OI7). Explaining Socially Responsible Consumer Behavior: A Meta-Analytic Review of Theory of Planned Behavior. Journal of International Consumer Marketing, 29(2), p. 9I-IO3. https://doi.org/Io.Io80/o896I530.20I6.1251870

Hughner, R. S., Mcdonagh, P., Prothero, A., Shultz, C. J., \& Stanton, J. (2007). Who are organic food consumers? A compilation and review of why people purchase organic food. Journal of Consumer Behaviour, 6(2), p. 94-IIO. https://doi.org/IO.IOO2/cb.2IO

Joshi, Y., \& Rahman, Z. (2OI5). Factors affecting green purchase behaviour and future research directions. International Strategic Management Review, 3(I/2), p. I28-I43. https://doi.org/ IO.IOI6/j.ism.20I5.04.OOI

Kaiser, F. G., Hübner, G., \& Bogner, F. X. (2005). Contrasting the Theory of Planned Behavior with the Value-Belief-Norm Model in Explaining Conservation Behavior. Journal of Applied Social Psychology, 35(IO), p. 2I50-2I7O. https://doi.org/IO.IIII/j.I559-I8I6.2005.tbo22I $3 \cdot \mathrm{X}$

Liobikienè, G., \& Bernatonienè, J. (2OI7). Why determinants of green purchase cannot be treated equally? The case of green cosmetics: Literature review. Journal of Cleaner Production, I62(I), IO9-I2O. https://doi.org/IO.IOI6/j.jclepro.2OI7.05.2O4

Nações Unidas (2015). Transforming our world: The 2030 Agenda for Sustainable Development. https://sustainabledevelopment.un.org/post2OI5/transformingourworld

Pearson, D., Henryks, J., \& Jones, H. (20II). Organic food: What we know (and do not know) about consumers. Renewable Agriculture and Food Systems, 26(2), p. I7I-I77. https:// doi.org/IO.IOI7/SI742I7O5IOOOO499

\section{Revista Administração em Diálogo}

ISSN 2178-0080 
Peattie, K. (2010). Green consumption: Behavior and norms. Annual Review of Environment and Resources, 35(I), p. 195-228. https://doi.org/Io.II46/annurev-environ-O32609-094328

Sahota, A. (2018). The global market for organic food and drink. In Willer, H., \& Lernoud J. (Eds.). The World of Organic Agriculture: Statistics and Emerging Trends 2018. Bonn, Research Institute of Organic Agriculture, Frick, and IFOAM - Organics International. https://www. organic-world.net/yearbook/yearbook-20I8/pdf.html

Sahota, A. (20I9). The global market for organic food and drink. In Willer, H., \& Lernoud, J. (Eds.) The World of Organic Agriculture: Statistics and Emerging Trends 2019. Bonn, Research Institute of Organic Agriculture, Frick, and IFOAM - Organics International. https:// orgprints.org/370I8/I/willer-lernoud-20i9-world-of-organic-low.pdf

Sampieri, R. H., Collado, C. F., \& Lucio, M.P. B. (20I3). Métodos de Pesquisa. Porto Alegre, RS: Penso.

Scalco, A., Noventa, S., Sartori, R., \& Ceschi, A. (2OI7). Predicting organic food consumption: A meta-analytic structural equation model based on the theory of planned behavior. Appetite, II2(I), p. 235-248. https://doi.org/ІІ.IOI6/j.appet.2OI7.02.007

Shaughnessy, J. J., Zechmeister, E. B., \& Zechmeister, J. S. (2O12). Metodologia de pesquisa em psicologia. Porto Alegre, RS: AMGH.

Souza, K. J. C., Filho, R. A. M., Santos, V. H. S., Melo, A. S., \& Cavalcanti, A. M. (2018). Perfil dos consumidores de alimentos orgânicos no Brasil. Sustentabilidade e Responsabilidade Social em Foco, 4(I), p. 87-99. https://poisson.com.br/20I8/produto/sustentabilidade-eresponsabilidade-social-em-foco-volume-4-2/

Willer, H., \& Lernoud, J. (Eds.) (2019a). The World of Organic Agriculture: Statistics and Emerging Trends 2019. Bonn, Research Institute of Organic Agriculture (FiBL), Frick, and IFOAM - Organics International. https://orgprints.org/37OI8/I/willer-lernoud-20I9world-of-organic-low.pdf

Willer, H., \& Lernoud, J (2orgb). Current Statistic on Organic Agriculture Worldwide: Area, Operators, and Market. In Willer, H., \& Lernoud, J. (Eds.) The World of Organic Agriculture: Statistics and Emerging Trends 2019. Bonn, Research Institute of Organic Agriculture (FiBL), Frick, and IFOAM - Organics International. https://orgprints.org/37oro/I/willer-lernoud -2Or9-world-of-organic-low.pdf

Willer, H., Lernoud, J, \& Kemper, L. (20I9). The World of Organic Agriculture 2org: Summary. In Willer, H., \& Lernoud, J. (Eds.) The World of Organic Agriculture: Statistics and Emerging Trends 2019. Bonn, Research Institute of Organic Agriculture (FiBL), Frick, and IFOAM - Organics International. https://orgprints.org/37OI8/I/willer-lernoud-2OI9world-of-organic-low.pdf 